\title{
MANIFESTAÇõES RESPIRATóRIAS NA MALÁRIA POR PLASMODIUM FALCIPARUM E VIVAX (1)
}

C. BOTEIHO (2), L. S. GUEDES BARBosa (2), J. L. AQUUNo (3), M. D. SILVA (4), S. M. P. MEIRELLES (5) \& J. R. B. JARDIM (6)

\section{R E S U M O}

Foram selecionardos 110 pacientes que procuraram a SUCAM/CUIABA para diagnóstico e tratamento de malária, com queixa de febre e procedente de área malarígena. Nestes pacientes foram realizados: gota espessa para a pesquisa de Plasmodium; aplicação de questionário padronizado para detectar sintomas respiratórios presentes no momento da entrevista; gasometria arterial e espirometria para avaliar a função pulmonar.

Desta amostra, 62 pacientes foram positivos para malária sendo 41 P. vivax, 20 P. falciparum e 01 forma mista (grupo Malárico); 48 mostraram-se negativos à pesquisa de hematozoários através da gota espessa (grupo Não Malárico).

As manifestações respiratórias encontradas nesta população quando comparadas os grupos Malárico e Não Malárico, foram respectivamente:

$1 .^{\circ}$ - Sintomáticos Respiratórios de $46,8 \%$ para $229 \%(\mathrm{p}<0,05)$;

2.) Alterações Espirométricas de 53,2\% para 20,8\% (p $<0,05)$;

3.) Alterações Gasométricas de $62,9 \%$ para $25,0 \%(\mathrm{p}<0,05)$.

Com estes resultados concluímos que há nítida associação entre a Malária produzida pelo $\mathbf{P}$. vivax e $\mathbf{P}$. falciparum e as manifestaçóes respiratórias apresentadas, nesta população de estudo.

UNITERMOS: Malária pulmonar; Malária — sintomas respiratórios.

\section{N T R O D U G $\mathbf{A} O$}

A malária é endêmica em todo o Estado de Mato Grosso e vem atingindo taxas elevadas nos últimos anos devido, principalmente, a manutenção de localidades com alto gráu de transmissíbilidade, como às da micro-região Norte-matogrossense, uma das três áreas mais importante de malária hiperendêmica do Brasil ${ }^{2 x}$. Um intenso e desorganizado fluxo $\mathrm{mi}$ gratório de individuos das mais variadas pro. cedências, dirigidas para os projetos de colo. nizações ou para os garimpos, faz com que a endemia transforme-se em grande risco de morbi-mortalidade para esses migrantes, quase sempre não imụnes.

O resultado disto é uma vasta gama de manifestações clínicas que a moléstia pode apresentar ao atingir diversos setores do or-

(1) Trabalho parcialmente financiado pelo CNPq - Processo n.० 40.7176/83.

(2) Professor Assistente do Departamento de Medicina da Universidade Federal de Mato Grosso, Cuiaba, Brasil.

(3) Professor Titular do Departamento de Medicina da Universidade Federal de Mato Grosso, Cuiabá, Brasil.

(4) Médico-bolsista do Projeto Polonoroeste/MT da Universidade Federal de Mato Grosso, Cuiabr, Brasil.

(5) Professor Adjunto-Doutor do Departamento de Medicina da Universidade Federal de Mato Grosso, Cuiaba, Brasil.

(6) Professor Adjunto-Doutor da Escola Paulista de Medicina, São Paulo, Erasil.

Endereço para correspondència: Dr. Clóvis Botelho. Av. Fernando Corrêa, s/n. UFMT/CCBS-I/NDS. CEP 78100 Cuisba, Mato Grosso, Prasil. 
BOTELHO, C.; GUEDES BARBOSA, L. S.; AQUINO, J. L.; SILVA, M. D.; MEIRELIES, S. M. P. \& JARDIM, J. R. B. - Manifestações respiratórias na malária por Plasmodium falciparum e vivạ. Rev. Inst. Med. trop. São Paulo, 29: 337-345, 1987.

ganismo, entre os quais o aparelho respiratório.

O envolvimento pulmonar na malária já é descrito na literatura e o aspecto clínico varia desde o acometimento do trato respiratório superior até o edema pulmonar $9,10,11,12$, $13,14,20,22$

As lesões ultra-estruturais que ocorrem nas membranas das hemácias parasitadas causam aglutinação e aderência ao endotélio vascular dos vasos profundos ${ }^{17,24}$. A hiperplasia do sistema reticulo-endotelial com atividade fagocitária aumentada na malária 28;34 levaria a obstrução de pequenos vasos e o apa. recimento de tromboses em vasculatura pulmonar 18,30. A presença de imunocomplexos circulantes 1 e sua deposição no epitélio alveo. lar 33 também são citadas como participes na gênese das alterações respiratórias encontradas nos indivíduos infectados. Alguns autores $3,6,8,19$ relatam um aumento da permeabilidade capilar pulmonar causada por uma ina. dequada compensação homeostática da vasodilatação generalizada que ocorre durante a fase aguda da malária. Outros mecanismos que levariam às desordens do aparelho respiratório são: disfunção hipotalâmica secundária ao metabolismo pertubado, causando espasmo venular pulmonar ${ }^{8}$; coagulação intravascular disseminada 14 e hiper-hidratação iatrogênica 6 .

De todas as explicações fisiopatologicas para as manifestações respiratórias na malária o denominador comum são as alterações na micro-circulação pulmonar. Acredita-se que estes fatores podem estar se somando, ora preponderanda um, ora outro, e que as repercussões sobre $o$ indivíduo dependerão muito do gráu da parasitemia, da sua imunidade, do tempo decorrido da infecção e da eficácia terapêutica empregada 4 .

O objetivo deste estudo é o de descrever alterações respiratórias em pacientes maláricos em atendimento ambulatorial. Com isto propõe-se dar uma contribuição para o esclarecimento das alterações respiratórias na malária, lembrando que para o diagnóstico precoce e uma terapêtica efetiva, devem ser conhecidas todas as formas de apresentação da doença.

\section{MATERIAL E METTODOS}

Uma população de 110 pacientes foi submetida ao estudo, sendo estes selecionados dentre os pacientes que procuraram a SUCAM/ CUIABÁ/MT para diagnóstico e tratamento de malária. Eles eram provenientes dos mais variados locais do Estado de Mato Grosso e de Rondônia e o tempo de estudo compreendeu o periodo de outubro de 1984 a julho de 1985. Todos os pacientes selecionados preencheram as seguintes características: idade entre 12 e 60 anos; não ter usado medicação prévia específica para malária até 1 (um) ano de antecedência; não ser portador de moléstia cárdio-pulmonar; disposição voluntária de participar do trabalho. A sequiência dos eventos foi: 1.a) Identificação; 2.a) Aplicação do questionário padronizado para detectar sintomas respiratórios; 3.a) Punção da polpa digital para gota espessa; 4. ${ }^{\text {a) }}$ Coleta de sangue arterial para gasometria 5.a) Realização de teste de função pulmonar (espirometria).

Foram considerados como sintomáticos respiratórios todos os indivíduos que apresentaram no momento da entrevista pelo menos um dos sintomas inquiridos: tosse, expectoracão, chiado e rouquiđão. O sintoma dispnéia ("falta de ar") foi retirado por ser passivel de confusão. Foram considerados não sintomá. ticos todos os individuos que negaram a presença de sintomas respiratórios. Foi considerado como tabagista $o$ indivíduo que tivesse feito uso do tabaco por periodo mínimo de 01 (um) ano, sob qualquer forma e como não tabagista, aos que negaram uso do tabaco por um periodo mínimo de 01 (um) ano.

A punção da polpa digital para realização da gota espessa foi feita pela equipe técnica da SUCAM, que em seguida corava as lâminas e fazia a leitura. O resultado era fornecido em termos da presença ou não de hematozoários e quando positivo determinava-se o tipo de Plasmodium encontrado, por técnica semiquantitativa, segundo os parâmetros da O.M.S. ${ }^{26}$.

Com o resultado fornecido pela equipe técnica da SUCAM, formou-se 2 grupos:

A) GRUPO MALARICO: Todos os pacientes com lâminas positivas para Malária. 
BOTEI,HO, C; GUEDES BARBOSA, L. S.; AQUINO, J. L.; SILVA, M. D.; MEIRELLES, S. M. P. \& JARDIM; J. R. B. - Manifestações respiratórias na malária por Plasmodium falciparum e vivax. Rev. Inst. Med. trop. São Paulo, 29:
337-345, 1987.

B) GRUPO NĀO MALARICO: Todos os pacientes que foram negativos para Malária.

A coleta do sangue arterial foi realizada pelo pesquisador clínico em artéria radial esquerda, após teste de perfusão capilar, com seringa descartável de 5cc heparizada. Após a coleta a amostra de sangue arterial era guardada sob gelo, dentro de caixa de isopor, para posterior análise no aparelho analisador de gases - Corning - Analyser Blood Gases - 165, que era calibrado no momento da rea. lização do exame. O tempo de permanência das amostras no recipiente com gelo não excedeu à 2 horas.

Para a classificação da pressão parcial de oxigênio no sangue arterial em valor normal, ou não, utilizou-se as equações de MELLENGAARD ${ }^{23}$ para os grupos etários de 15 a 40 anos e de 41 a 75 anos, sendo o valor médio e desvio padrão de cada grupo discriminados abaixo:

$$
\begin{aligned}
& 15-40 \text { anos: } 96,7 \pm 6,2 \mathrm{mmHg} \\
& 41-75 \text { anos: } 89,4 \pm 8,0 \mathrm{mmHg}
\end{aligned}
$$

Como limite inferior da normalidade da $\mathrm{PaO}_{2}$ utilizou-se o valor médio do grupo subtraído do valor do desvio padrão multiplicado por 1,64 , o que corresponde a $86,5 \mathrm{mmHg}$ para o grupo mais jovèm e $76,3 \mathrm{~mm}$ para o mais idoso. Uma vez que Cuiabá tem uma altitude aproximada de 180 metros não se utilizou fator de correção para a pressão barométrica local nas equações citadas.

A prova espirométrica foi realizada no Espirógrafo Vitatrace 130 e seguiu-se as recomendaçōes da American Thoracic Society para as mensurações dos parâmetros espirométricos $^{2}$. Com os parâmetros estudados, capacidade vital forçada (CVF), volume espiratório forçado no $1 n^{\circ}$ segundo ( $\mathrm{VEF}_{1}$ ), fluxo expiratório forçado 25-75\% (FFF 25-75\%) e a relação $\mathrm{VEF}_{1} / \mathrm{CVF}$ classificou-se os pacientes em relação à sua função pulmonar, em normais ou como tendo insuficiência pulmonar ventilatória do tipo obstrutivo, restritivo ou misto.

Para a análise estatística foi utilizada o teste do $\mathrm{x}^{2}$ nos casos em que se procurou verificar a possibilidade de associação entre os variáveis sintomas respiratórios e espirometria com a infecção malárica. Utilizou-se a participação de Cochran nas tabelas com mais de 1 grau de liberdade. Foi aplicado o teste " $t$ " de Student pars verificar a diferença dos valores médios da $\mathrm{PaO}_{2}$. $\mathrm{O}$ nível de significância foi estabelecido em $5 \%(\alpha=0,05)$.

\section{RESULTADOS}

A população de estudo, no total de 110 pacientes teve uma média de idade de $31,1 \pm$ 11,0 anos sendo constituída por 101 indivíduos do sexo masculino e 9 do sexo feminino. A distribuição dos indivíduos pertencentes ao estudo foi feita em 2 grupos: A) Malárico: 62 pacientes ( 41 P. vivax, 20 P. falciparum e 1 forma mista - vivax e falciparum); B) Năo Malárico: 48 pacientes.

A procedência dos pacientes obedeceu aos fluxos migratórios já existentes no Estado de Mato Grosso, vindo das microregiôes: Norte Matogrossense, 50,9\%; Baixada Cuiabana, 27,3\%; Alto Paraguai, 3,6\% e do Estado de Rondônia, 18,2\%.

A relação entre o grau da parasitemia no momento da avaliação pulmonar e as alterações nela encontradas estão nas tabelas I (P. vivax) e II (P. falciparum). Com o aumento da parasitemia notamos maior prevalência de comprometimento pulmonar nos individuos infectados pelo $P$. falciparum, o que não ocorreu com os infectados pelo P. vivax.

A prevalência de sintomáticos respirató. rios no grupo Malárico foi de $46,8 \%$, não existindo diferença significativa entre as duas espécies parasitárias. A estatística mostrou as. sociação entre sintomas respiratórios e infecção causada pelo $P$. falciparum $\left(x^{2}\right.$ cal $=$ $\left.6,61^{*}\right)$ a pelo $P$. vivax $\left(x^{2}\right.$ cal $\left.=4,41^{*}\right)$. A prevalência do grupo Não Malárico foi de $22,9 \%$ (tabela III).

A espirometria mostrou que alteraçōes de ventilação pulmonar ocorreram com maior freqữênca no grupo Malárico $(53,2 \%)$, não existindo diferença significativa entre as duas espécies parasitárias. A análise do $\mathrm{x}^{2}$ mostrou assaciação entre alterações espirométricas e infecção causada pelo P. falciparum ( $x^{2}$ cal = $14,96 \%$ *) e pelo $P$. vivax ( $x^{2}$ cal $\left.=5,45^{*}\right)$. No 
BOTEU.FO, C.; GUEDES BARBOSA, L. S.; AQUINO, J. L.; SILVA, M. D.; METRELIES, S. M. P. \& JARDIM, J. R. B. - Manifestações respiratórias na malária por Plasmodium falciparum o vivax. Rev. Inst. Med. trop. São Paulo, 29: 337-345, 1987.

T A B E L A I

Distribuição do grupo Malárico (P. vivax) em relação aos diferentes níveis de avaliação respiratória, segundo o grau de parasitemia.

\begin{tabular}{|c|c|c|c|c|c|c|}
\hline \multirow{3}{*}{$\begin{array}{l}\text { Parasitemia } \\
\text { (P. vivax) }\end{array}$} & \multicolumn{2}{|c|}{$\begin{array}{c}\text { Sintomas } \\
\text { Respiratórios }\end{array}$} & \multicolumn{2}{|c|}{ Espirometria } & \multicolumn{2}{|c|}{$\mathrm{PaO}_{2}$} \\
\hline & Sim & Não & Alterada & Normal & Alterada & Normal \\
\hline & N.० \% & N. $\%$ & N. $\%$ & N.o $\%$ & N.O \% & N. $\%$ \\
\hline+ & $8(47,1)$ & $9(52,9)$ & $7(41,2)$ & $10(58,8)$ & $12(70,6)$ & $5(59,4)$ \\
\hline++ & $6(42,8)$ & $8(57,2)$ & $7(50,0)$ & $7(50,0)$ & $6(42,8)$ & $8(57,2)$ \\
\hline$+1+$ & $4(40,0)$ & $6(60,0)$ & $4(40,0)$ & $6(60,0)$ & $8(80,0)$ & $2(20,0)$ \\
\hline Total & $18(43,9)$ & $23(56,1)$ & $18(43,9)$ & $23(56,1)$ & $27(65,8)$ & $14(34,2)$ \\
\hline
\end{tabular}

T A B E I A II

Distribuição do grupo Malárico (P. falciparum) em relação aos diferentes níveis de avaliação respiratória, segundo o grau de parasitemia.

\begin{tabular}{|c|c|c|c|c|c|c|}
\hline \multirow{3}{*}{$\begin{array}{c}\text { Parasitemia } \\
\text { (P. falciparum) }\end{array}$} & \multicolumn{2}{|c|}{$\begin{array}{l}\text { Sintomas } \\
\text { Respiratórios }\end{array}$} & \multicolumn{2}{|c|}{ Espirometria } & \multicolumn{2}{|c|}{$\mathrm{PaO}_{2}$} \\
\hline & Sim & Não & Alterada & Normal & Alterada & Normal \\
\hline & N. $\%$ & N.\% \% & N.。 $\%$ & N. $\%$ & N.o $\%$ & N.०\% \\
\hline+ & $7(50,0)$ & $7(50,7)$ & $9(64,3)$ & $5(35,7)$ & $6(42,8)$ & $8(57,2)$ \\
\hline+1 & $2(50,0)$ & $2(50,0)$ & $3(75,0)$ & $1(25,0)$ & $4(100)$ & - \\
\hline$+1+$ & $2(100)$ & - & $2(100)$ & - & $2(100)$ & - \\
\hline Total & $11(55,0)$ & $9(45,0)$ & $14(70,0)$ & $6(30,0)$ & $12(60,0)$ & $8(40,0)$ \\
\hline
\end{tabular}

T A B E L A III

Distribuiçăo dos sintomáticos e não sintomáticos respiratórios na população de estuđo.

\begin{tabular}{|c|c|c|c|c|c|c|c|}
\hline \multirow{2}{*}{\multicolumn{2}{|c|}{ Grupos }} & \multicolumn{2}{|c|}{$\begin{array}{l}\text { Sintomáticos } \\
\text { Respiratórios }\end{array}$} & \multicolumn{2}{|c|}{$\begin{array}{l}\text { Não Sintomáticos } \\
\text { Respiratórios }\end{array}$} & \multicolumn{2}{|c|}{ Total } \\
\hline & & N. ${ }^{\circ}$ & $\%$ & N.o & $\%$ & N.o & $\%$ \\
\hline A. & ${ }_{\text {Malárico }}$ & 29 & $(46,8)$ & 33 & $(53,2)$ & 62 & (100) \\
\hline & P. vivax & 18 & $(43,9)$ & 23 & $(56,1)$ & 41 & (100) \\
\hline & P. falclparum & 11 & $(55,0)$ & 9 & $(45,0)$ & 20 & (100) \\
\hline & $F+v$ & - & - & 1 & $(100)$ & 1 & (100) \\
\hline B. & Não Malárico & 11 & $(22,9)$ & 37 & $(77,1)$ & 48 & (100) \\
\hline & Total & 40 & $(36,4)$ & 70 & $(63,6)$ & 110 & $(100)$ \\
\hline
\end{tabular}

axbxc $\mathrm{x}^{2} \cdot$ cal $=7,74^{*} \mathrm{p}<0,05$

$(a+b) \times c x^{2}$ cal $=7,09 * p<0,05$

axo $x^{2}$ cal $=0,05 \mathrm{NS}$

grupo Não Malárico as alterações de ventila. ção pulmonar foi de $20,8 \%$. A obstrução ao fluxo aéreo foi o distúrbio ventilatório mais encontrado, 38,7\% no grupo Malárico e $18,7 \%$ no grupo Não Malárico (tabela IV). Quanto ao fluxo espiratório forçado 25-75\% (FFF 2575\%), no grupo malárico $62,9 \%$ tinham valores abaixo de $80 \%$ do valor previsto, não exis. tindo diferença signifficativa entre as duas especies parasitárias. Existe associação entre in. fecção causada pelo $P$. falciparum $\left(x^{2}\right.$ cal $=$ 10,81*) e pelo P. vivax $\left(x^{2}\right.$ cal $\left.=8,99^{*}\right)$ com os velores abaixo de $80 \%$ do valor previsto para o FEF 25-75\% (tabela V).

A comparação das médias dos valores $\mathrm{PaO}_{2}$ mostra que nos indivíduos com faixa etária até 40 anos do grupo Malárico, é significativamente menor que a dos individuos do grupo Não Malárico. Na faixa acima de 40 
BOTELHO, C.; GUEDES BARBOSA, L. S.; AQUINO, J. L.: SILVA, M. D.; MEIREILLES, S. M. P. \& JARDIM, J. R. B. - Manifestações respiratórias na malária por Plasmodium lalciparum e vivax. Rev. Inst. Med. trop. São Paulo, 29:
337-345, 1987.

T A B E I A TV

Distribuição dos resultados da espirometria na população de estudo

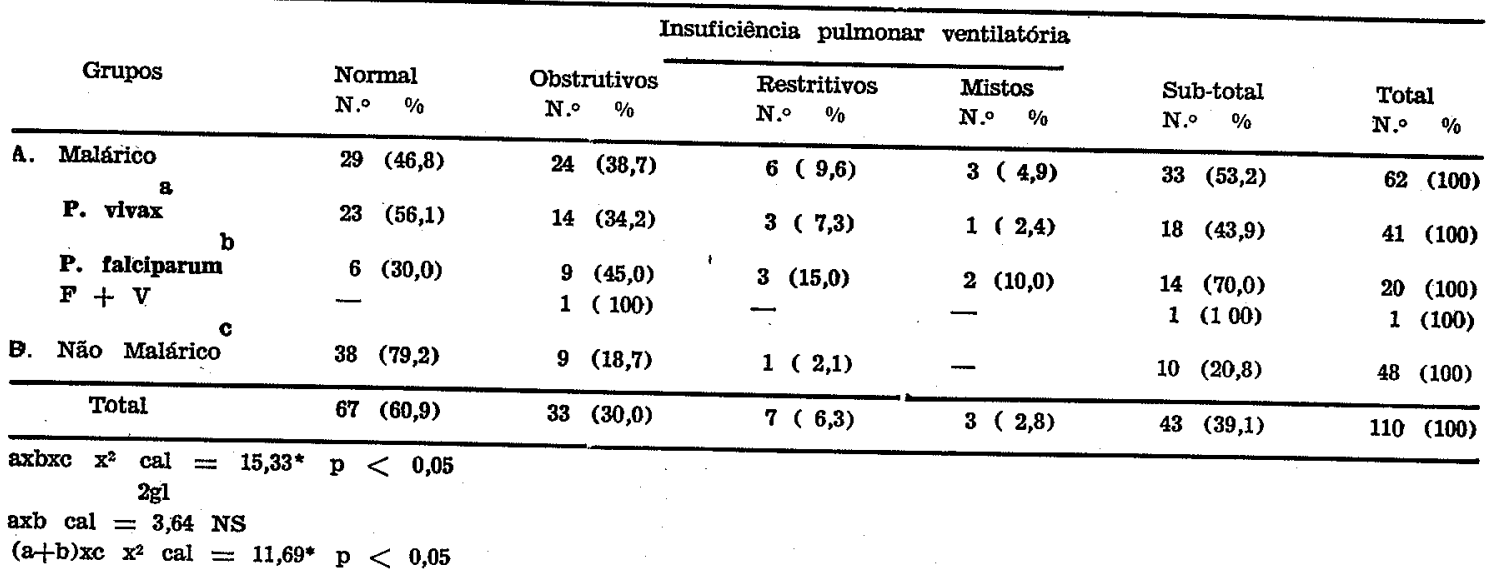

T A E E I A

Distribuiçāo dos valores do fluxo expiratório forçado 29 - $75 \%$ na população de estudo

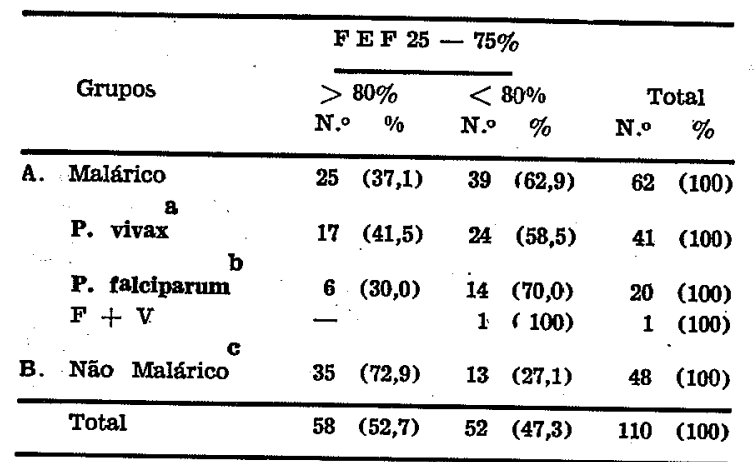

${ }_{\mathrm{FEF}_{23}}-75 \%=$ Fluxo Expiratório Florçado 25-75\%

axbxc $x^{2}$ cal $=14,04^{*} p<0,05$ $2 \mathrm{gl}$

axb $x^{2}$ cal $=0,72$ NS

$(\mathrm{axb}) \times \mathrm{x}^{2}$ cal $=13,32^{*} \mathrm{p}<0,005$

anos não existe diferença significativa entre as médias dos valores da $\mathrm{PaO}_{2}$ dos indivíduos Malárico e Não Malárico. Nã̃o existe diferen. çá significativa entre as espécies parasitárias (tabela VI).

Se agruparmos todos os indivíduos com $\mathrm{PaO}_{2}$ abaixo dos valores previstos para a ida. de (acima e abaixo do limite inferior da normalidade) observamos que no grupo Malárico há $39(62,9 \%)$ ao passo que no grupo Não Malárico há somente $12(25 \%)$. Se considerarmos somente os valores da $\mathrm{PaO}_{2}$ abaixo do limite inferior da normalidade, que seriam os pacientes com hipoxemia sem qualquer dúvida, ainda assim o grupo Malárico registra
T A B E I A VI

Distributição da média dos valores da $\mathrm{PaO}_{2}$, na população de estudo

\begin{tabular}{|c|c|c|c|c|}
\hline \multirow{2}{*}{$\begin{array}{c}\text { Faixa Etária } \\
\text { (anos) }\end{array}$} & \multirow{3}{*}{$\frac{\text { P. vivax }}{31}$} & \multirow{3}{*}{$\frac{\frac{\text { Malaria }}{\text { P. falciparum }}}{15}$} & \multirow{3}{*}{$\frac{\text { Sub-Total }}{46}$} & \multirow{3}{*}{ Não Málárico } \\
\hline & & & & \\
\hline $15-40 n$ & & & & \\
\hline$x$ & 91,94 & 92,81 & $92,22^{a}$ & $96,86^{a}$ \\
\hline d.p. & 10,59 & 7,28 & 9,56 & 7,53 \\
\hline+40 & 10 & 5 & 16 & 10 \\
\hline $\begin{array}{r}\mathbf{x} \\
\text { d.p. }\end{array}$ & 91,37 & 85,5 & $\begin{array}{r}b 7,77 \\
076\end{array}$ & $91,77^{\mathbf{b}}$ \\
\hline u.p. & 14,01 & 1,40 & 9,76 & 11,36 \\
\hline
\end{tabular}

a $t_{\mathrm{cal}}=-2,490,01<\mathrm{p}<0,02$ (significativo)

b. $t_{\text {cal }}^{c a l}=0,92 p>0,005$ (não significativo)

um número maior de pacientes $14(22,6 \%)$ do que o grupo Não Malárico $14(8,3)$.

$O$ fato do individuo fumar não alterou as suas chances de pertencer a um grupo ou outro, o que seria esperado. Assim, dos 60 fumantes, 39 estavam no grupo Malárico e 29 no Não Malárico. Dos 50 não fumantes, 23 eram do grupo que teve malária e 27 não ti. veram malária, A observação do gráfico 1 mostra claramente que as alteraçōes da $\mathrm{PaO}_{2}$ foram basicamente decorrentes da malária e não do cigarro. Dos indivíduos não fumantes, $19,2 \%$ deles não tiveram malária mas o seu $\mathrm{PaO}_{2}$ estava abaixo da média e/ou do limite inferior previsto para a idade; em contraposição doś não fumantes, no grupo Malárico, 52\% tinha a $\mathrm{PaO}_{2}$ diminuída. A mesma proporção ocorre no grupo de fumantes, isto $E$, dos que 
BOTELHO, C.; GUEDES BARBOSA, L. S.; AQUINO, J. L.; SILVA, M. D.; MEIRELIES, S. M. P. \& JARDIM, J. R. B. - Manifestações respiratórias na malária por Plasmodium falciparum e vivax. Rev. Inst. Med. trop. São Paulo, 29: $337-345,1987$.

não tinham maláris $33 \%$ deles tinham a $\mathrm{PaO}_{2}$ diminuída mas este valor subia para $85,7 \%$ no grupo Malárico.

\section{DISCUSSÃO}

A sede da Diretoria Regional da SUCAM de Mato Grosso, em Cuiabá, recebe diariamen. te numerosos pacientes para diagnóstico e tratamento da malária, sendo que a maioria deles o tratamento é realizado a nível ambulatorial. Os casos mais graves são encaminhados a rede hospitalar da cidade ficando, muitas vezes, sob orientação do pessoal técnico daquele órgão.

Na população de estudo houve predominância do sexo masculino $(91,8 \%)$, que representa a força de trabalho das regiões de procedência dos indivíduos, sendo que a maioria deles vieram da micro-região Norte Matogrossense, onde a não fixação do homem à terra, dificuldade de acesso aos serviços de saúde e a estrutura sócio-econômica, contribui para a manutenção da endemia e colabora com a reintrodução da malária em outros Estados ${ }^{21,32}$.

Notamos também um grande número de pacientes procedentes de Rondônia (18,2\%), traduzindo o fluxo migratório existente entre aquele estado e a capital matogrossense lem. brando que com isto está formado o corredor para a introdução de casos em outros estados (casos importados), principalmente para os da região Sul e Sudeste do País. Para se tentar impedir que tal fato ocorra, que é um risco já bastante conhecido, seria necessário maior integração de todos os órgãos envolvidos direta e indiretamente com a problemáti. ca da malária no Brasil.

Ao ser analisado o gráu de parasitemia no comprometimento pulmonar notamos que somente para o P. falciparum houve um maior percentual de individuos com sintomas respiratórios, alterações espirométricas e gasométricas quando aumentou a parasitemia. Tal fato não foi observado nos indivíduos infectados pelo $\mathbf{P}$. vivax, aqui as alterações encontradas não guardam relação com o aumento da parasitemia.

A prevalência de sintomáticos respiratórios foi maior no grupo Malárico, não existindo đi- ferença significativa entre a ação do Plasma dium falciparum ou vivax nas manifestações dos sintomas analisados. Os sintomas mais encontrados foram: tosse, expectoração, chia. do e rouquidão. BUCK et al 7 no Hospital San Lázaro, Manila, Philipinas, estudaram 1000 casos de Malária $(56 \%$ P. falciparum, $38 \%$ P. vivax, 6\% infecção mista) e mostraram que os sintomas respiratórios apresentados por muitos pacientes são passiveis de confusão, pois na admissão hospitalar desses casos estudados em $11,3 \%$ diagnosticou-se influenza e em $1,3 \%$, pneumonia. Não houve detalhamento de qual espécie parasitária foi maior a participação nas alterações respiratórias encontradas e não se discutiu os mecanismos envolvidos. LARA et al ${ }^{16}$ estudando um grupo de 15 gestantes infectadas pelo $P$. falciparum observaram que $93 \%$ do seus pacientes apre. sentaram tosse não produtiva, não comentando a razão do fenômeno. Por outro lado, OLIVEIRA et al 25 fazendo revisão do quadro clínico em 163 pacientes portadores de malária por $\mathbf{P}$. falciparum, mostraram que $7,9 \%$ destes apresentaram sintomas respiratórios, sendo todos eles não portadores de moléstia pulmonar prévia, e sugerindo que substâncias vasoativas são liberadas na malária provocando alteraçães na musculatura brônquica. Não há relato neste trabalho se os sintomas foram inquiridos sob forma de questionário dirigido para o aparelho respiratório, o que dificulta a comparação com os nossos dados. A prevalência dos sintomas respiratórios no grupo Não Malárico (22,9\%) foi semelhante ao encontrado por outros autores $5,15,28$, quando são estudados grupos populacionais usando o mesmo método (sintomas referidos).

As alterações espirométricas encontradas foram mais prevalentes no grupo Malárico $(53,2 \%)$ do que no grupo Não Malárico $(20,8 \%)$ e o distúrbio ventilatório mais comum foi a obstrução ao fluxo aéreo, demonstrando com isso transtornos na mecânica brônquica, impedindo a livre passagem de ar através das vias aéreas. Quando analisamos somente o FEF 25-75\%, que é um bom índice para estudo da permeabilidade das pequenas vias aéreas ${ }^{27}$, notamos que houve diminuição do fluxo nos dois grupos estudados sendo esta diminuição, entretanto, muito mais acentuada no grupo Malárico (62,9\% dos pacientes) do que no gru- 
BOTELHO. C.; GIEDES BARBOSA, I. S.; AQUTNO, J. L.; SILVA, M. D.; MEIRELIES, S. M. P. \& JARDTM, J. R. B. - Manifestações respiratórias na malária por Plasmodium falciparum $\theta$ vivax. Rev. Inst. Med. trop. São Paulo. 29:
$337-345,1987$.

po Não Malárico (27,1\% dos pacientes). Este fato mostra a possibilidade das pequenas vias aéreas serem um local de acometimento pela malária. TONG et al 31 já haviam mostrado que testes de função pulmonar podem estar anormais em pacientes com malária pelo $\mathbf{P}$. falciparum, mesmo na ausência de evidência clínica ou radiológica de complicação pulmonar, podendo persistir esta alteração até o 9. dia da terapêutica. No presente estudo as duas espécies parasitárias causaram alterações na mecânica brônquica, em graus variáveis, detectados pelos índices espirométricos estudados.

Para avaliarmos melhor os resultados obtidos com a espirometria seria interessante se conseguíssemos repetir todo o protocolo inicial após cura clínica da infecção malárica. A comparação pré e pós tratamento não nos foi possível realizar devido às dificuldades inerentes ao próprio tipo de pacientes por nós estudados (atendimento ambulatorial, pacientes em trânsito etc.).

A grande diferença no percentual de alterações da $\mathrm{PaO}_{2}$ dos grupos Maláricos e Não Malárico, mostra que deve ter ocorrido distúr. bios na estrutura pulmonar dos pacientes com malária. $O$ fato do grupo por nós estudado ser jovem afasta a possibilidade dessas alterações poderem ser devidas à bronquite crônica, enfisema ou moléstia tumoral. Mesmo insistindo-se na chance de ocorrência dessas doenças deve-se ter em mente que o grupo Não Malárico tinha, praticamente, a mesma média de idade $(30,6 \pm 10,6$ anos $)$ que o grupo Malárico (31,7 $\pm 11,2$ anos).

A análise do hábito de fumar nos dois grupos e as alterações da $\mathrm{PaO}_{2}$ deixa, também, evidente que a malária é a responsável por esta diminuição da oxigenação arterial (gráfico 1). Um outro fator que pode ser responsável pelo decréscimo na saturação do oxigênio é o quadro febril que os pacientes apre sentavam no momento da avaliação gasométri. ca e a sua presença, nos dois grupos estudados, evidencia ainda mais as alterações da $\mathrm{PaO}_{2}$ pela malária.

Como já dito anteriormente para a espirometria, o ideal seria a repetição do estudo da gasometria arterial após o período de cura
GRAFICO 1

ALTERAÇÕS DE $P_{0} O_{2}$ e tabagismo, NA POPULACAO DO ESTADO

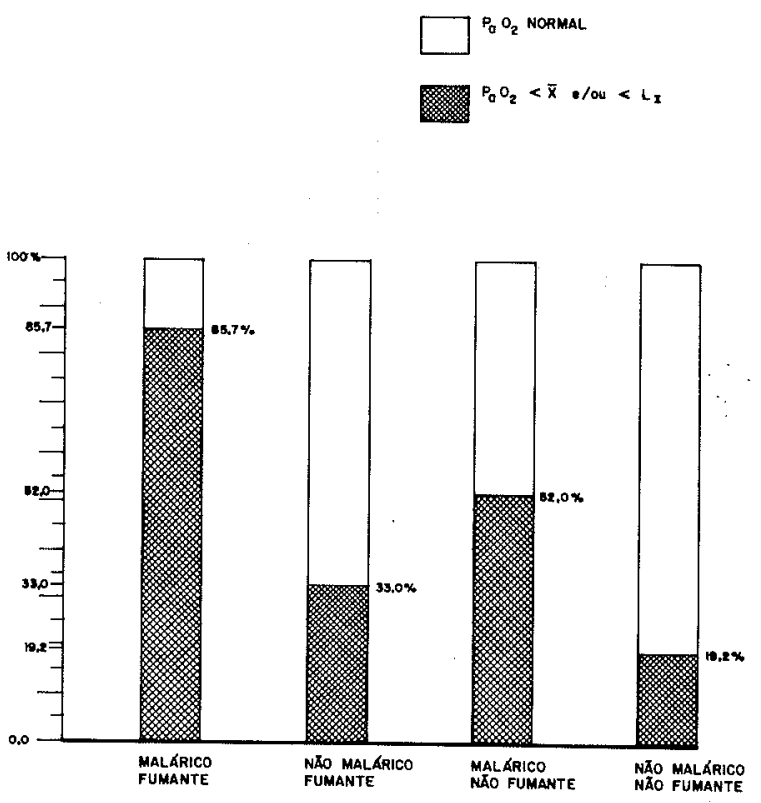

$<\bar{X}=$ AEAIXO DO VALOR MÉOIO PREVISTO

$\angle L_{\mathbf{I}}=$ ABAIXO DO LIMITE INFERIOR DE NORMALIOADE

para que possa afirmar, categoricamente, que essas alterações eram provenientes do acometimento pulmonar pela malária. Mas mesmo na impossibilidade de um novo exame, as evidências são muito importantes no sentido que tal acometimento pulmonar esteja ocorrendo.

A ação do tabagismo no aparelho respiratório já encontra-se bem estabelecida por vários autores 5,28 e a sua influência no aparecimento das alterações respiratórias encontradas não pode deixar de ser considerada. 0 hábito tabágico apareceu em $54,5 \%$ dos indivíduos estudados e a análise estatística empregada $\left(x^{2}\right)$ mostrou associação entre ele e a variável sintomáticos respiratórios $(\mathrm{p}<0,01)$. Separando os grupos, Malárico e Não Malárico, para efetuarmos o controle da variável tabagismo na presença de sintomas respirató. rios e usando o mesmo teste estatístico, notamos que esta associação persistiu no grupo 
BOThLHO, C.; GUEDES BARBOSA, L. S.; AQUINO, J. L.; SILVA, M. D.; MEIRELIES, S. M. P. \& JARDIM, J. R. B. - Manifestações respiratórias na malária por Plasmodium falciparum e vivax. Rev. Inst. Med. trop. São Paulo, 29: 337-345, 1987.

Não Malárico ( $p<0,05)$. Entretanto, no grupo Malárico tal associação deixou de existir ( $p>0,05$ ), mostrando com isso uma independência entre as variáveis tabagismo e sintomáticos respiratórios, para o intervalo de confiança por nós delimitado $(\alpha=5)$. Acreditamos que houve somação dos efeitos do tabaco e a infecção malárica sobre $o$ aparelho respiratório, nesta população de estudo.

As alterações respiratórias detectadas no presente estudo sugerem que a malária por P. falciparum e P. vivax mesmo nos casos leves (tratamento ambulatorial), leva a um aumento da permeabilidade capilar produzindo edema de mucosa, liberação de substâncias vasoativas e transtornos a nível da membrana alveolo-capilar.

Salienta-se a necessidade de serem estimulados estudos clínicos ou experimentais para o total esclarecimento das manifestações respiratórias da malária.

\section{SUMMARY}

Malaria respiratory manifestations by Plàsmomodium falciparum and $P$. vivax.

A group of 110 patients (101 male, 9 female with mean age $\pm S D: 31,1 \pm 11,0$ years) with fever, coming from an endemic malaria region, was studied at SUCAM-CUIABA, for diagnosis and treatment of possible protozoal infection.

For this group a simplified questionnaire on respiratory symptoms was applied and laboratory tests consisting of peripheral blood film, arterial blool gas analysis and pulmonary function test were done. 62 patients had a positive test of malaria (41 P. vivax, $20 \mathrm{P}$. falciparum, $1 \mathrm{~F}+\mathrm{V}$ ) and 48 were negative.

\section{THE FOLLOWING RESULTS WERE OBSERVED:}

+ Malaria -

\begin{tabular}{lrr}
\hline Respiratory symptoms & $46,8 \%$ & $22,9 \%$ \\
Abnormal pulmonary function & & \\
test & $53,2 \%$ & $20,8 \%$ \\
Abnormal blood gas analysis & $62,9 \%$ & $25,0 \%$ \\
\hline
\end{tabular}

We conclude that there is an association between malarial infection and the presence of respiratory problems.

\section{AGRADECIMENTOS}

Ao pessoal técnico do Laboratório Central da Diretoria Regional da SUCAM/MT.

Ao Prof. Antonio Rufino Neto e Prof. Odécio Sanchez, da USP - Ribeirão Preto, pela orientação metodológica e estatística.

A Prof.a Marta Duarte de Barros (UFMT) pela revisão do manuscrito.

\section{REFERENCIAS BIBLIOGRAFICAS}

1. ADAM, C.; GENITEAU, M.; GOUGEROT-POCIDALO, M.; BERROUT, P.; LABRAS, J.; GHBERT, C. \& MOREL-MAROGER, L. - Cryoglobulins, cjrculation immunecomplexes and complement activation cerebral malaria. Infect. Immun., 31: 530-535, 1981.

2. AMERICAN THORACIC SOCIETY. Snowbird Worlshop on standardization on Spirometry. Amer. Rev. resp. Dis., 119: 831-840, 1979.

3. BLANLOEIL, Y.; BARON, D.; LAJARTRE, A. Y. \& NICOLAS, F. - Syndrome de détresse respiratoire aiguë de l'adulte au cours de l'accès pernicieux palustre. A propos d'un cas. Sem: Hôp. Paris, 56: 1088-1090, 1980.

4. BOTELHO, C.; GUEDES EARBOSA, L. S.; RIBEIRO, C. L. S. \& PINTO, G. R. - Manifestações pulmonares da malária por P. falciparum - a propósito de dois casos. J. Pneumol., 11: 202-205, 1985.

5. BOTELHO, C.; FONTES, H. M.; GUEDES BARBOSA, L. S.; BORGE, A. N. \& MEIRELLES, S: M. P. Araputanga/MT. Prevalência de tabagismo o sua correlação com os sintomáticos respiratórios. J. Pneumol., 10: 105, 1984.

6. BROOKS, M. H.; KIEL, F. W.; SHEEHTY, T. W. \& BARREY, K. G. - Acute pulmonary edema, in falciparum malaria. New Engl. J. Med., 279: 732-737, 1968.

7. BUCK, R. L.; ALCANTARA, A. K.; UYLANGCO, C. v. \& CROSS, J. H. - Malaria at San Lazaro Hospital, Manila, Philippines, 1979-1981. Amer. J. trop. Med. Hyg., 32: 212-216, 1983.

8. CAYEA, P. D.; RUBIN, E. \& TEIXIDOR, H. S. Atypical pulmonary malaria. Amer. J. Roentgenol., 137: 51-55, 1981.

9. DEATON, J. G. - Fatal pulmonary edema as a com plication of acute falciparum malaria. Amer. J. trop. Med. Hyg., 19: 196-201, 1970.

10. FEIN, A.; RACKOW, E. C. \& SHAPIRO, L. - Acute pulmonary edema in $P$. falciparum malaria. Amer. Rev. resp. Dis., 118: 425-429, 1978. 
BOTELHO, C.; GUEDES BARBOSA, L. S.; AQUINO, J. L.; SILVA, M. D.; MEIRELLES, S. M. P. \& JARDIM, J. R. B. - Manifestações respiratórias na malária por Plasmodium talçparum e vivax. Rev. Inst. Med. trop. São Paulo, 29: 337-345, 1987.

11. FLETCEFR, J. R.; BUTLER, T.; KOPRIVA, C. J. \& RATLIF, J. L. - Acute Plasmodium falciparum malaria. Arch. intern. Med., 120: 617-619, 1972.

12. GASCARD, E.; RAOUZAD, M. \& ROQUE, G. - Accés puniciaux palustre à forme pulmonary. Marseille-méd., 105: 713-718, 1968.

13. GODARD, J. E. \& HANSEN, R. A. - Interstitial pulmonary edema in acute malaria. Radiology, 101: 523-524, 1971.

14. GCODAL, H. B. - Giant nuclear masses in the hungs and blood in malignant malaria. Lancet, 17: 1124. 1126, 1973,

15. GUEDES BARBOSA, L. S.; BOTHLFO, C.; FORTES, H. M.; BORGES, A. N.; FORTES, F. M. \& MEI. REI,LES, S. M. P. - Morbidade por exames elínicos na região do Polonoroeste/MT, 1983. Revista da UFMT, 3: $317-319,1984$.

16. LARA, R. M.; LEGES, D. M.; SANTANA, C. H. \& CETARD, N. - Paludismo y embarajo. Rev. méd. dominic., 43: 34-37, 1983.

17. LUSE, S. A. \& MHLLER, L. H. - Plasmodium talciparum malaria. Amer. J. trop. Med. Hyg., 20: $655-660,1971$.

18. MAcCALLUM, D. K. - Pulmonary changes resulting from experimental malaria infection in hamsters. Arch. Path., 86: 681-688, 1968.

19. MALLOY, J. P.; BROOKS, M. H. \& BARRY, K. G. - Pathophysiology acute falciparum of malaria. II fluid compartmentalization. Amer. J. Med., 43: 745$750 ; 1967$.

20. MARKS, S. M.; HOLLAND, S. \& GELFAND, M. Malarial lung: report of a case from Africa - successfully treated with intermittent positive ventilation. Amer. J. trop. Yed. Hyg., 26: 179-180, 1977.

21. MARQUES, A. C. \& PINHEIRO, E. A. - Fluxos de casos de malária no Brasil em 1980. Rev. bras. Malar., 34: 1-31, 1982.

22. MARTFUL, R. W.; KAILFMMBACH, J. \& ZWI, S. Pulmonary edema in falciparum malaria. Brit. med. J., 30: 1763-1764, 1979.

23. MELLENGAARD, K. - The alveolar-arterial oxygen difference: its size and components in normal man. Acta physiol. scand., 67: 10-20, 1966.
24. MULLER, L. H.; USAMI, S. \& CHIEN, S. - Altera. ction in the rheologic properties of P. knowlesi infected red cells. A possible mechanism for capillary obstruction. J. clin. Invest., 50: 145-151, 1971.

25. OLIVEIRA, R. M. G. \& SOUZA, I. M. - Revisão do quadro clinico em pacientes portadores de maĺria por P. faleiparum. Rev. bras. Malar., 35: 57-63, 1983.

26. OPS/OMS. Manual de diagnóstico microscópico da maläria. 4.ed. Washington, O:P.S./O.M.S., 1975. (Publ. Cient. n.o 276).

27. RATTO, O. R.; BOGOSSIAN, M. \& SANTOS, M. L. Insuficiência respiratória. São Paulo, Livraria Atheneu, 1981. Cap. 3, p. 62-66.

28. RUFINO NETTO, A. \& CARVALFIEIRO, J. R. Doenças do aparelho respiratório e doenças infecciosas e parasitárias na morbidade referida pela população, Ribeirão Preto, SP. - $1975 . \quad$ Medicina (Ribeiräo Preto), 15: 79-83, 1982.

29. SHEAGREN, J. N.; TOBIE, J. E.; FOX, L. M. \& WOLFF, S. M. - Reticuloendothelial systen phagocytic function in nturaly acquired human malaria. J. Lab. clin. Med., 75: 481-485, 1970.

30. SPANGLER, W. L.; GRIBBE; D.; ABIIDGOARD, C. \& HARRISON, J. - Plasmodium knowlesi malária in the Rheusus monkey. Vet. Path., 15: 83-91, 1978.

31. TONG, M. J.; BAILANTINE, T. V. N. \& YONEL, D. B. - Pulmonary function studies in $\mathbf{P}$. falciparum malaria. Amer. Rev. resp. Dis., 106: 23-29, 1972.

32. WANDERLEY, D. M. V.; ANDRADE, J. C. R.; NENEGUITTI, L. C.; CHINEILATTO, M. J. \& DUTRA, A. P. - Malária no Estado de São Paulo, Brasil, 1980 a 1983. Rev. Saúde públ. (S. Paulo), 19: 28-32, 1985.

33. WEISS, M. L. - Immune complexes in the lungs of mice infected with Plasmodium berghel. Israel J. med. Sci., 14: 655-658, 1978:

34. ZIESSMAN, H. A. - Lung Uptake of $99 \mathrm{Tc}$ - Sulfur colloid in falciparum malaria: case report. J. nucl. Med., 17: 794-796, 1976.

Recebido para pủblicação em $17 / 3 / 1987$. 